Article

\title{
Variation of the Performance of Machine-Learning Based Image Classifier in Automated Detection of Itch-Induced Scratch
}

\author{
Chuan $\mathrm{Liu}^{1,2,+}$, Sheng-Xiang Yan ${ }^{1,+}$, Xiao-Bo Wu${ }^{1}$, Zhi-Jun Zhang ${ }^{3}$, Wei Li ${ }^{4 *}$ (1) \\ 1 Institute of Special Environmental Medicine, Institute of Pain Medicine, Nantong University, No. 9, Seyuan \\ Road, Nantong City, Jiangsu Province, P. R. China \\ 2 College of Medicine and Health, Lishui University, No. 1, Xueyuan Road, Lishui City, Zhejiang province, P. \\ R. China \\ 3 School of Medicine, Nantong University, No. 19, Qixiu Road, Nantong City, Jiangsu Province, P. R. China \\ 4 Institute of Special Environmental Medicine, Institute of Nautical Medicine, Nantong University, No. 9, \\ Seyuan Road, Nantong City, Jiangsu Province, P. R. China \\ * Correspondence: wli148@aucklanduni.ac.nz; \\ + These authors contributed equally to this work.
}

\begin{abstract}
A 'little brother' of pain, itch is an unpleasant sensation that creates a specific urge to scratch. To date, various machine-learning based image classifiers (MBICs) have been proposed for quantitative analysis of itch-induced scratch behaviour of laboratory animals in an automated, non-invasive, inexpensive and real-time manner. In spite of MBICs' advantages, the overall performances (accuracy, sensitivity and specificity) of current MBIC approaches remains inconsistent, with their values varying from $\sim 50 \%$ to $\sim 99 \%$, for which the reasons underlying have yet to be investigated further, both computationally and experimentally. To look into the variation of the performance of MBICs in automated detection of itch-induced scratch, this article focuses on the experimental data recording step, and reports here for the first time that MBICs' overall performance is inextricably linked to the sharpness of experimentally recorded video of laboratory animal scratch behaviour. This article furthermore demonstrates for the first time that a linearly correlated relationship exists between video sharpness and overall performance (accuracy and specificity, but not sensitivity) of MBICs, and highlight the primary role of experimental data recording in rapid, accurate and consistent quantitative assessment of laboratory animal itch.
\end{abstract}

Keywords: Itch; Scratch; Automated real-time detection; Machine-learning based image classifier; Image sharpness

\section{Introduction}

Itch is a sensation that causes the desire to scratch [1-3]. In laboratory animal studies, scratch has been used as an objective correlate for quantitative assessment of itch [4-7]. Usually, scratch is counted by human annotators by playing the video back and forth, which is a time-consuming and laborious process with inherent subjectivity in quantitative assessment of laboratory animal itch. To this end, several machine-learning base experimental approaches have been developed to address this issue [8-10]. Very recently, Park and his colleagues [11] reported an automated real-time approach using machine learning-based image classifier (MBIC), which can be of practical use for the quantitative analysis of mouse scratching recorded with commercially available video cameras. This MBIC approach is non-invasive, inexpensive and suitable for objective counting of scratching with 
overall accuracy similar to or better than existing automated counting methods [11-13]. Moreover, it requires neither an attachment nor a surgical implantation of a device to mouse [11].

Nonetheless, MBICs' overall performances to date still remains inconsistent, including accuracy, sensitivity and specificity [11]. On average, the two-step decision tree (2DT) MBIC approach [11] performs with a sensitivity and specificity of $95.19 \%$ and $92.96 \%$, respectively. While its sensitivity ranges from $94.8 \%$ to $99.1 \%$, its specificity possesses a wider range from $68.6 \%$ to $85.1 \%$, as reported by Park and his colleagues [11]. As for other MBIC approaches, such as support vector machine (SVM), k-nearest neighbour ( $\mathrm{kNN})$, convolutional neural network $(\mathrm{CNN})$, recurrent neural network (RNN) and long short-term memory (LSTM), their sensitivities and specificities possess a even wider range from $55 \%$ to $97 \%$ [11]. In addition to the inconsistency of MBICs' performances, the reasons underlying have yet to be investigated further, both computationally and experimentally. Since various computational MBIC approaches have been reported already [8], and essentially all machine learning methods possesses an inherent trade-off between specificity and sensitivity that is controlled through the classification threshold, this article therefore chooses to focus on the experimental data recording step, i.e., the primary wet-lab step before any step(s) computational, and aims to investigate the relationship(s) between the MBICs' overall performances and the sharpness of the laboratory animal scratch video (LASV).

\section{Materials and Methods}

\subsection{Chemicals and laboratory animals}

Compound 48/80 (C48/80, Sigma, USA) is a polymer that provokes itch histamine-dependently in both human and mice [14]. Prior to use, Compound 48/80 was dissolved in $150 \mathrm{mM}$ saline (Sichuan Kelun Pharmaceutical Co., Ltd, Sichuan Province, China) and stored at $4^{\circ}$.

All animal-related experimental procedures were approved by Animal Ethical Committee of Nantong University (Jiangsu Province, China). Adult male health C57BL/6 mouse (weight: $24 \pm 2 \mathrm{~g}$ ) were purchased from Laboratory Animal Center of Nantong University, Jiangsu Province, China. All mice were housed singly and were maintained on a $12 / 12$ day /night cycle at $22-25^{\circ}$ with free access to rodent food and water in environmentally controlled specific pathogen-free (SPF) conditions.

\subsection{Video recording, processing and preliminary analysis}

Before LASV recording, a C57BL/6 mouse was placed into a box (dimensions $16 \mathrm{~cm} \times 14 \mathrm{~cm} \times 15$ $\mathrm{cm}$, Figure 1) for 15 minutes to ensure their familiarity with the box environment. Afterwards, the box (Figure 1) was cleaned with 70\% ethanol and distilled deionized water. Of the LASV recording process, the general experimental setting is illustrated in Figure 1 briefly as below.

1. First, C57BL/6 mice were anesthetized and placed in the box (Figure 1) before an neck-subcutaneous injection of $50 \mu \mathrm{lC} 48 / 80$ at $2 \mathrm{mg} / \mathrm{ml}$.

2. A digital video camera recorder Sony ${ }^{\mathrm{TM}}$ HDR-CX405 (Sony Corporation, Japan) was employed to record a set of LASVs (time duration: 40 to 60 minutes) at 25 frames per second (fps) with a $1280 \times 720$ pixel resolution, assuming that the camera is fast enough to capture all scratching behaviors of C57BL/ 6 mouse $[4,15]$. By default, the focus is adjusted automatically by the Sony ${ }^{\mathrm{TM}}$ HDR-CX405 camera.

3. Each C57BL/ 6 mouse scratch was counted first by human annotators by playing LASVs back and forth, also recorded manually were the time labels (i.e., the beginning point and the ending point) of each set of scratch movements of the C57BL/ 6 mouse.

4. All LASVs were splitted into a series of image sequences with the FFmpeg command at $10 \mathrm{fps}$ on an in-house Ubuntu machine.

5. With a set of in-house python scripts, all LASV images were linked to their respective time labels and manual annotations, i.e., scratch or non-scratch, for the subsequent training step of the machine-learning image classification processes. 


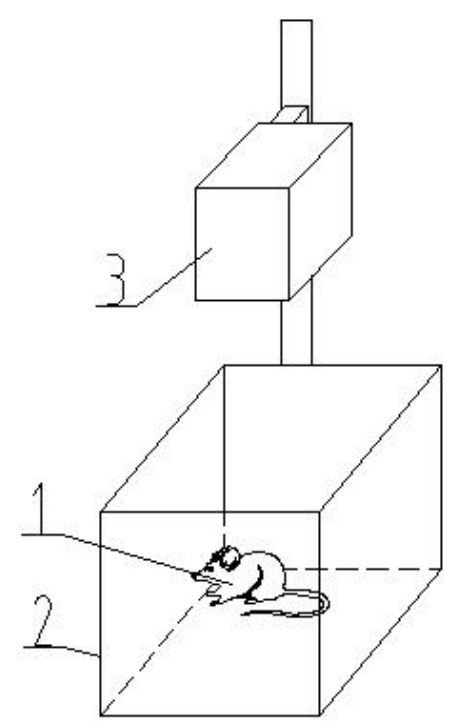

Figure 1. A minimalist sketch of the experimental setting for LASV recording. In this figure, labels 1, 2 and 3 represent the C57BL/ 6 mouse, the box and the position of the video-recording camera, respectively.

6. The sharpness of every LASV image was quantified as the variance of its Laplacian (supplementary file detect_blur.py).

Given that image sharpness is the focus here, it is necessary to ensure a reasonable scale coverage of the LASV sharpness, representing at least LASVs with high, medium and low degrees of sharpness. Therefore, we manually selected five from all experimentally recorded LASVs, labelled them according to their original file names, namely 305 (high sharpness), 306 (median sharpness), 309 (low sharpness), 310 (low sharpness) and 311 (high sharpness), respectively (Table 1).

\subsection{Keras MBIC image classification}

In total, 135769 LASV images were extracted from the manually selected five videos (Table 1). First, the 135769 images were pooled into one directory, and subsequently grouped into two sub-directories according to human annotations (i.e., scratch or non-scratch), with one sub-directory named scratch and another named nonscratch, respectively. For the MBIC's training step, 13650 LASV images (representing scratch) were imported into the open source MBIC software package (implemented in Python with Keras [11]), along with 13650 LASV images (representing non-scratch) which were randomly selected from the rest of all LASV images. The whole Keras MBIC software package is submitted as a supplementary file, along with its two main python scripts for MBIC image classification (Table 2), for which further details of the training and testing steps are included in Tables 2 and 1.

Of all 135769 computationally classified LASV images (Table 2), each and every image was subsequently linked to its time label, human annotation (scratch or non-scratch) and automated MBIC annotation (scratch or non-scratch), too. With both manual and computational annotations in place, a set of in-house python scripts were employed to statistically analyze the overall performances of the Keras MBIC approach and their potential relationships with the sharpness of experimentally recorded

LASVs. 


\begin{tabular}{|l|c|c|c|c|c|c|}
\hline Video number & 305 & 306 & 309 & 310 & 311 & Total \\
\hline Total number of images (X1) & 25542 & 27659 & 27020 & 28268 & 27280 & 135769 \\
\hline Trained Scratch (X2) & 330 & 2350 & 3860 & 5820 & 1290 & 13650 \\
\hline Trained Non Scratch (X3) & 2786 & 2834 & 2644 & 2526 & 2860 & 13650 \\
\hline Trained Total (X4=X2+X3) & 3116 & 5184 & 6504 & 8346 & 4150 & 27300 \\
\hline Trained Scratch Ratio (X2/X1) & 0.0129 & 0.0849 & 0.1428 & 0.2058 & 0.0472 & 0.1005 \\
\hline Trained Non-Scratch Ratio (X3/X1) & 0.1091 & 0.1027 & 0.0978 & 0.0893 & 0.1048 & 0.1005 \\
\hline Trained Total Ratio (X4/X1) & 0.1220 & 0.1876 & 0.2406 & 0.2951 & 0.1520 & 0.2010 \\
\hline C48/80 injection & No & Yes & Yes & Yes & Yes & Yes \\
\hline
\end{tabular}

Table 1. Statistics of the LASV images used for the training step of machine-learning based image classification of the five LASVs. For video $305,150 \mathrm{mM}$ saline was injected subcutaneously to the neck of C57BL/ 6 .

\begin{tabular}{|c|l|l|l|}
\hline Step & Input & Python script & Output \\
\hline Training & 27300 images & train_network.py & scratch_not_scratch.model \\
\hline Testing & all 135769 images & test_network.py & scratch or non-scratch \\
\hline
\end{tabular}

Table 2. Training and testing of the machine-learning based image classification. For the training step, half of the 27300 images were with the manual annotation scratch, the other half non-scratch. In this table, all python scripts are submitted as supplementary files.

\section{Results}

\subsection{Quantification of the sharpness of LASVS}

With a preliminary visual inspection of the five LASVs, their sharpness (or blurrinesses) did vary in a manner of both inter- and intra-video, which is briefly illustrated in Figure 2 with two LASV images.
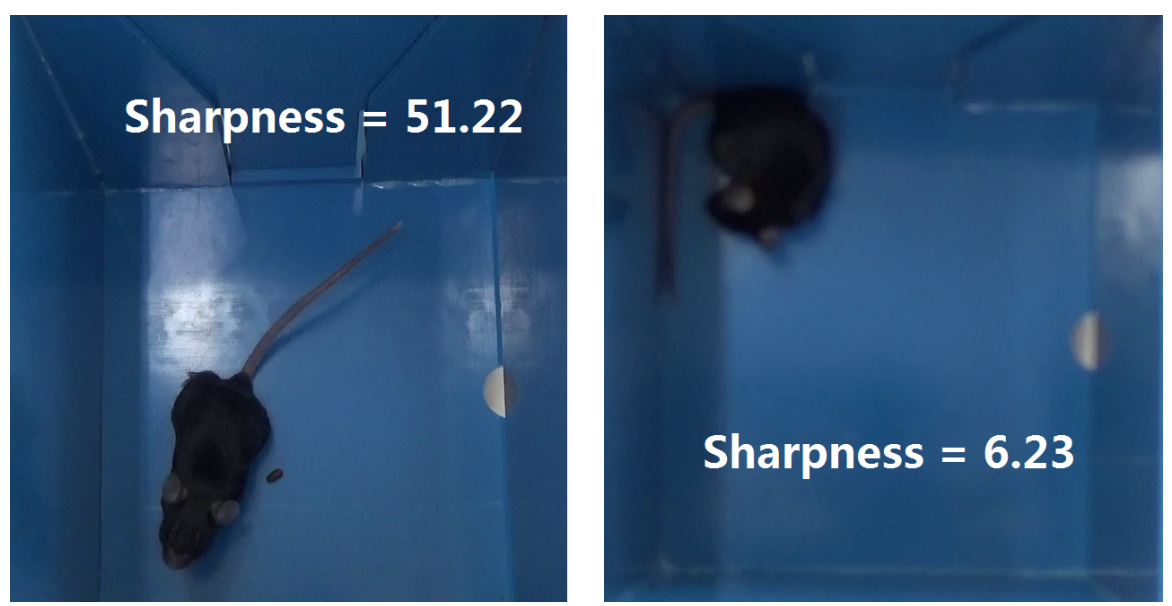

Figure 2. One LASV image with high degree (51.22, left) of sharpness (file name is 311out4381.png) and one LASV image with low degree (6.23, right) of sharpness (file name is 306out11856.png). Here, supplementary file detect_blur.py was used to quantify the sharpness of the two LASV images. The original versions of the two cropped and edited LASV images are submitted in supplementary file supplementary.pdf.

In addition to Figure 2, Table 3 presents a quantitative statistical analysis of the sharpness of the five LASVs, a necessary step for further investigation into its relationship(s) with the Keras MBIC's overall performances, which are to be described and discussed below in details. 


\begin{tabular}{|c|c|c|c|c|c|}
\hline Video name & Average & Standard deviation & Maximum & Minimum & Sharpness \\
\hline 305 & 44.38 & 3.56 & 67.27 & 5.27 & High \\
\hline 306 & 34.10 & 3.13 & 53.01 & 6.23 & Median \\
\hline 309 & 29.91 & 2.67 & 37.66 & 6.95 & Low \\
\hline 310 & 28.26 & 3.08 & 37.04 & 8.11 & Low \\
\hline 311 & 41.47 & 2.16 & 58.91 & 12.28 & High \\
\hline
\end{tabular}

Table 3. A statistical analysis of the sharpness of the five LASVs. In this table, the sharpness of each video was quantified as the average and the standard deviation of the sharpness of all 135769 images extracted from the five LASVs, the sharpness of each image was quantified as the variance of its Laplacian.

\subsection{Overall performance of the Keras MBIC approach}

Among all 135769 computationally classified LASV images (Table 2), there were a total of 108146 $(96100+12046$, Tables 4) images where the computational annotation is the same as its manual counterpart, with an overall accuracy at $79.65 \%$. With a further video-specific analysis (Tables 4 ), it turned out that

1. The Keras MBIC approach here performed with accuracies ranging from $57.55 \%$ to $97.87 \%$.

2. The Keras MBIC approach here performed with sensitivities ranging from $61.52 \%$ to $95.58 \%$.

3. The Keras MBIC approach here performed with specificities ranging from $48.44 \%$ to $98.35 \%$.

Overall, these findings align well with the performances of the previously reported MBIC approaches [11], where a range of 55\% - 97\% was reported for their specificities and the sensitivities of the Keras MBIC approach here.

\begin{tabular}{|l|c|c|c|c|c|c|}
\hline Video number & 305 & 306 & 309 & 310 & 311 & Total/Average \\
\hline Scratch Test True (X5) & 203 & 2100 & 3587 & 5394 & 762 & 12046 \\
\hline Scratch Test False (X6) & 127 & 250 & 273 & 426 & 528 & 1604 \\
\hline Scratch Test Total (X7=X5+X6) & 330 & 2350 & 3860 & 5820 & 1290 & 13650 \\
\hline Non-Scratch Test True (X8) & 24796 & 21465 & 13653 & 10874 & 25312 & 96100 \\
\hline Non-Scratch Test False (X9) & 416 & 3844 & 9507 & 11574 & 678 & 26019 \\
\hline Non-Scratch Test Total (X10=X8+X9) & 25212 & 25309 & 23160 & 22448 & 25990 & 122119 \\
\hline Sensitivity (X5/X7) & 0.6152 & 0.8936 & 0.9293 & 0.9268 & 0.9558 & 0.8824 \\
\hline Specificity (X8/X10) & 0.9835 & 0.8481 & 0.5895 & 0.4844 & 0.9739 & 0.7869 \\
\hline Accuracy ((X5+X8)/X1) & 0.9787 & 0.8520 & 0.638 & 0.5755 & 0.9558 & 0.7965 \\
\hline
\end{tabular}

Table 4. Overall performance of the Keras MBIC approach. In this table, X1 is the same as in Tables 4.

To sum up, this table is combined with Table 4 as Table 1 in supplementary material supplementary.pdf.

With these quantitative video-specific analysis of the Keras MBIC approach's performances in place, it is now possible to further investigate their potential relationships with LASV sharpness. Here, with a set of in-house python scripts, the overall accuracy, the overall sensitivity and the overall specificity of the five LASVs were plotted against their respective sharpness, as shown in Figures 3, 4 and 5.

Specifically, Figures 3 and 5 demonstrate that the LASV sharpness is a linear correlate of both accuracy and specificity of the Keras MBIC approach here, with two Pearson's correlation coefficients $95.54 \%$ and $94.38 \%$, respectively. On the other hand, no linear correlation was detected between LASV sharpness and the sensitivity of the Keras MBIC approach here, as shown in Figure 4.

To test the importance of specificity in the overall performance of the Keras MBIC approach here, we plotted its accuracy against its specificity and found a strong linear correlation between them with a Pearson's correlation coefficient of 99.84\%, as shown in Figure 6. 


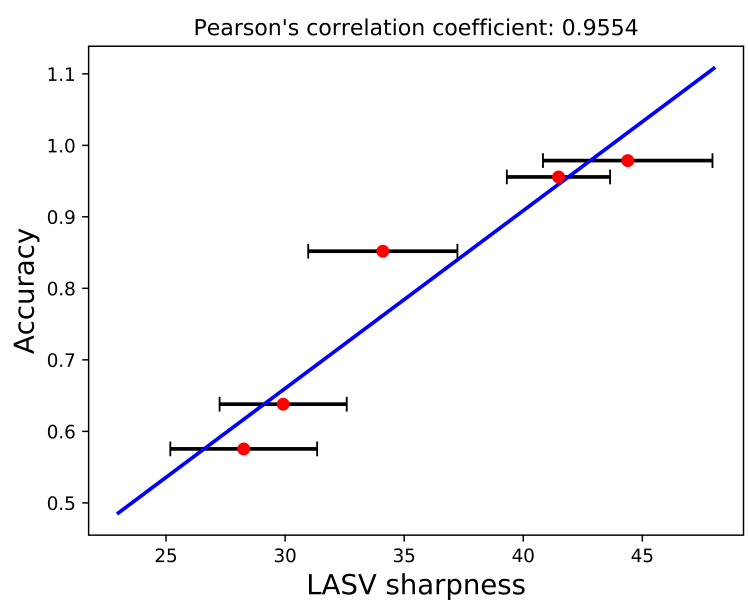

Figure 3. LASV sharpness influences the accuracy of the Keras MBIC approach in a linearly correlated manner.

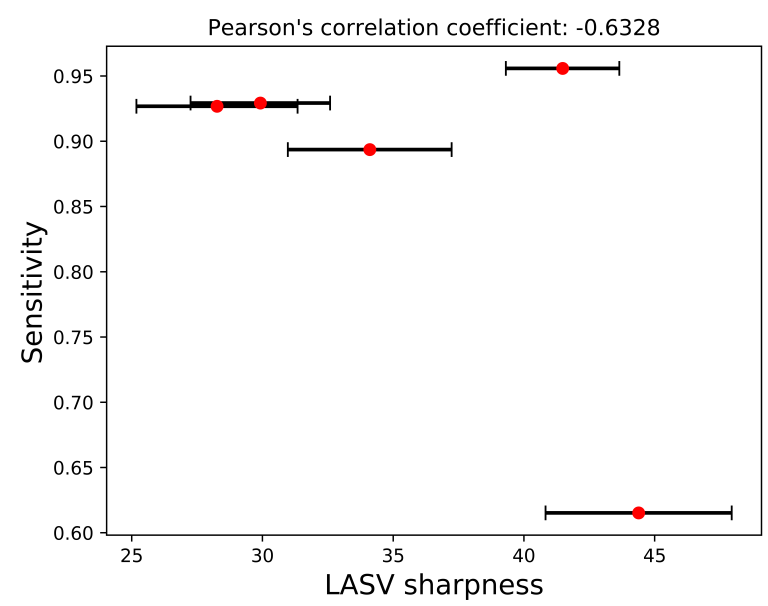

Figure 4. LASV sharpness does not influence the sensitivity of the Keras MBIC approach.

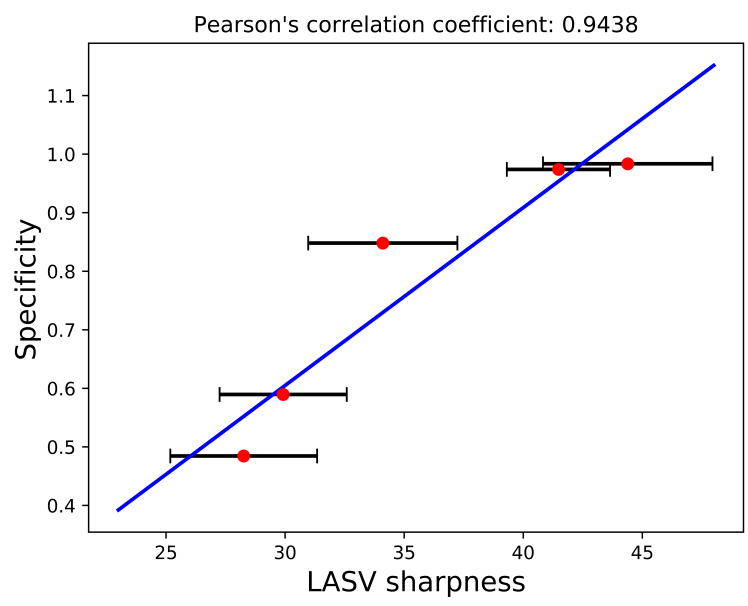

Figure 5. LASV sharpness influences the specificity of the Keras MBIC approach in a linearly correlated manner. 


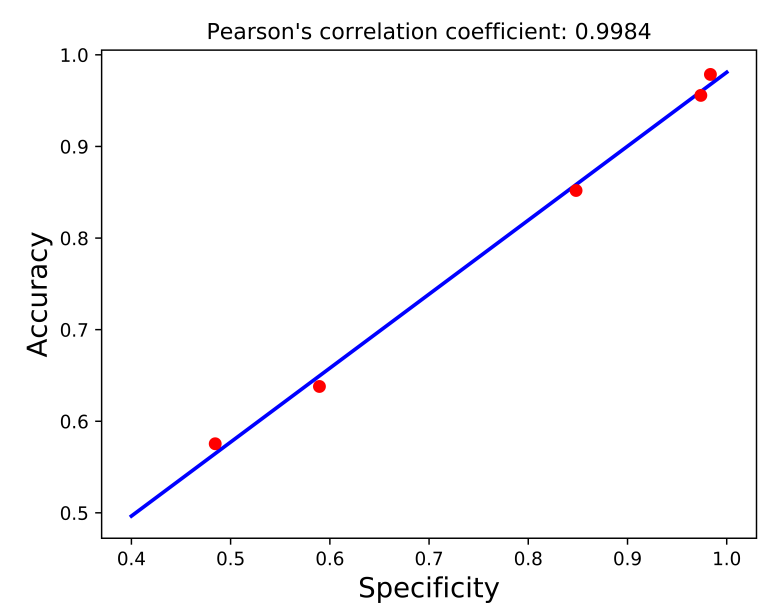

Figure 6. A linear correlation of the specificity and the accuracy of the Keras MBIC approach.

\section{Variation of the performance of MBICs in automated detection of itch-induced scratch: an experimental perspective}

While it has been already previously demonstrated that MBIC is a useful tool for automated quantitative assessment of laboratory animal itch [11], and on the fundamental and practical points of view, it also appears true that MBIC accuracy comes with LASV sharpness, no direct experimental study was reported yet as of January 17, 2020 to investigate the relationship between the two factors (i.e., accuracy and LASV sharpness) in a quantitative manner for the use of MBIC in automated detection of itch-induced scratch. Of the Keras MBIC approach here, this article puts forward a set of experimental evidences and reports for the first time that

1. LASV sharpness is a linear correlate of its accuracy.

2. LASV sharpness is not a linear correlate of its sensitivity.

3. LASV sharpness is a linear correlate of its specificity.

4. A linear correlation was observed between its specificity and accuracy.

5. No linear correlation was observed between its sensitivity and accuracy.

In addition to accuracy, sensitivity and specificity of the overall performance of MBICs, this article also highlights the importance of reproducibility and consistency of the overall performance of MBICs, which are just as important in rapid and accurate analysis of scratching for preclinical studies and high throughput drug screening for diseases like atopic dermatitis [16,17].

\section{LASV blurriness: why and what to do next?}

As discussed above, this article focuses on the primary wet-lab step, for which the experimental recording of LASVs is the weakest link in the loop. With a close naked-eye inspection of the five LASVs, we ask this questions: what makes the sharpness of all 135769 LASV images different from one another, both intra- and inter-video, as shown in Figure 2 and Table 3? To answer itr, we eyed on the experimental data recording step again, and boiled it down to two things: initial experimental setting and auto-focus of the video recording camera, for the accuracy and the precision of the focus of the video camera on C57BL/ 6 mouse, respectively.

1. The initial experimental setting (Figure 1) for LASV recording is fundamentally involved in the image quality of LASVs,

(a) because the video camera must be told accurately where to focus first before LASV recording, otherwise it will be difficult for the camera to achieve satisfactory focus on the subject (i.e., C57BL/ 6 mouse), and the camera therefore might give up on the automated adjustment 
of the focus, resulting in failed auto-focus [18]. That is, the initial experimental setting (Figure 1) needs to be as accurate as possible, as it is the first step of LASV recording, hence the primary determinant for LASV sharpness.

(b) Therefore, with the statistical analysis and the discussion above, we call for the establishment of a universal standard for the initial experimental setting, including all necessary parameters, such as the size and the color of the box (Figure 1), the position of the camera (Figure 1), the manufacturer of the camera, LASV recording frequency, LASV image resolution/size, etc. With this universal standard in place, it becomes possible to compare the results obtained with video sequences of varying (but constant) qualities to clearly determine the minimum level of LASV sharpness to apply MBIC to detect itch-induced scratch.

(c) In the long run furthermore, with such a universal standard, the MBIC training step will produce a universally applicable scratch_not_scratch.model (Table 2),too, as an input for the MBIC testing step directly, and thereby strengthening the efficiency and the consistency and the reproducibility of MBIC-based automated detection of itch-induced scratch $[19,20]$.

2. The focus of Sony ${ }^{\mathrm{TM}}$ HDR-CX405 on C57BL/ 6 mouse is also inextricably linked to the image quality of LASVs, as by default the focus is adjusted automatically by the Sony ${ }^{\mathrm{TM}}$ HDR-CX405 video camera. In general, there are four factors affecting the auto-focus performance of a camera: light level (level of illumination), subject contrast, camera motion and subject motion. Moreover, these factors are not independent; in other words, one may be able to achieve autofocus even for a dimly lit subject if that same subject also has extreme contrast, or vice versa [18].

(a) For LASV recording, we suggest an anti-vibration [7] disturbance-free laboratory area with appropriate light level, where no laboratory member walks by the box and the camera (Figure 1) every now and then, to avoid light level variation and enhance the autofocus performance of the video camera. Furthermore, we also suggest the use of appropriate LASV recorders and video acquisition settings in the application of MBIC in automated detection of itch-induced scratch.

(b) Supplementary file animate.gif included a truncated LASV built with three consecutive frames extracted from video 311, namely 311out85.png, 311out86.png and 311out87.png, representing the image of laboratory animal at $8.5,8.6$ and 8.8 seconds, respectively. The 0.3-second-long animate.gif is a clear representation that when a mouse scratches, it stands on one of its hind paws and uses another one of its hind paws to scratch its neck where C48/80 was injected, and that the subject contrast is strong enough for both naked-eye and MBIC to tell whether the mouse is scratching its neck or not at certain time point.

(c) As shown in Figure 1, the position of the camera is set from the beginning, camera motion is therefore not an issue for the Keras MBIC approach here.

(d) Mouse scratching can happen at ten times per second or more [3,6], which is a rather fast movement compared to the time (usually a fraction of a second [18]) necessary for one automated adjustment of the focus by the video camera. While most commonly available $30 \mathrm{fps}$ video cameras are equipped with image sensors fast enough to clearly record these scratch behaviour $[15,18]$, chances are that those recorded LASV images blur because the mouse scratches its neck too fast for the video camera to achieve a satisfactory focus on the subject and to clearly record each and every scratch movement of the mouse. To address this subject motion issue, further hardware improvements might be necessary for the autofocus sensor of LASV recording cameras.

Acknowledgments: We thank the National Supercomputing Center in Wuxi (Jiangsu Province, China) for both training and testing with the Keras software package for machine-learning based image classifications.

Author Contributions: Conceptualization, W.L.; methodology, W.L.; software, W.L.; validation, W.L.; formal analysis, Z.Z., W.L.; investigation, C.L. and S.Y.; resources, W.L.; data curation, W.L.; writing-original draft preparation, W.L.; writing-review and editing, X.W., W.L.; visualization, W.L.; supervision, W.L.; project administration, W.L.; funding acquisition, not applicable. 
Funding: This research received no external funding.

Conflicts of Interest: The author declares no conflict of interest.

1. Nie, Y.; Ishii, I.; Yamamoto, K.; Orito, K.; Matsuda, H. Real-time scratching behavior quantification system for laboratory mice using high-speed vision. Journal of Real-Time Image Processing 2009, 4, 181-190. doi:10.1007/s11554-009-0111-7.

2. Namer, B.; Reeh, P. Scratching an itch. Nature Neuroscience 2013, 16, 117-118. doi:10.1038/nn.3316.

3. Elliott, P.; G'Sell, M.; Snyder, L.M.; Ross, S.E.; Ventura, V. Automated acoustic detection of mouse scratching. PLOS ONE 2017, 12, e0179662. doi:10.1371/journal.pone.0179662.

4. Ishii, I.; Kurozumi, S.; Orito, K.; Matsuda, H. Automatic Scratching Pattern Detection for Laboratory Mice Using High-Speed Video Images. IEEE Transactions on Automation Science and Engineering 2008, 5, 176-182. doi:10.1109/tase.2007.902868.

5. Ehling, S.; Butler, A.; Thi, S.; Ghashghaei, H.T.; Bäumer, W. To scratch an itch: Establishing a mouse model to determine active brain areas involved in acute histaminergic itch. IBRO Reports 2018, 5, 67-73. doi:10.1016/j.ibror.2018.10.002.

6. Orito, K.; Chida, Y.; Fujisawa, C.; Arkwright, P.; Matsuda, H. A new analytical system for quantification scratching behaviour in mice. British Journal of Dermatology 2004, 150, 33-38. doi:10.1111/j.1365-2133.2004.05744.x.

7. Brash, H.; McQueen, D.; Christie, D.; Bell, J.; Bond, S.; Rees, J. A repetitive movement detector used for automatic monitoring and quantification of scratching in mice. Journal of Neuroscience Methods 2005, 142, 107-114. doi:10.1016/j.jneumeth.2004.08.001.

8. Smith, M.P.; Ly, K.; Thibodeaux, Q.; Weerasinghe, T.; Wu, J.J.; Yosipovitch, G.; Bhutani, T.; Liao, W. Emerging Methods to Objectively Assess Pruritus in Atopic Dermatitis. Dermatology and Therapy 2019, 9, 407-420. doi:10.1007/s13555-019-0312-3.

9. Salem, G.; Krynitsky, J.; Hayes, M.; Pohida, T.; Burgos-Artizzu, X. Three-Dimensional Pose Estimation for Laboratory Mouse From Monocular Images. IEEE Transactions on Image Processing 2019, 28, 4273-4287. doi:10.1109/tip.2019.2908796.

10. Tarrasón, G.; Carcasona, C.; Eichhorn, P.; Pérez, B.; Gavaldà, A.; Godessart, N. Characterization of the chloroquine-induced mouse model of pruritus using an automated behavioural system. Experimental Dermatology 2017, 26, 1105-1111. doi:10.1111/exd.13392.

11. Park, I.; Lee, K.; Bishayee, K.; Jeon, H.J.; Lee, H.; Lee, U. Machine-Learning Based Automatic and Real-time Detection of Mouse Scratching Behaviors. Experimental Neurobiology 2019, 28, 54. doi:10.5607/en.2019.28.1.54.

12. Reich, A.; Szepietowski, J.C. Diagnostic Procedures of Itch. In Itch-Management in Clinical Practice; S. Karger AG, 2016; pp. 24-28. doi:10.1159/000446013.

13. Su, X.Y.; Chen, M.; Yuan, Y.; Li, Y.; Guo, S.S.; Luo, H.Q.; Huang, C.; Sun, W.; Li, Y.; Zhu, M.X.; Liu, M.G.; $\mathrm{Hu}, \mathrm{J} . ; \mathrm{Xu}, \mathrm{T} . L$. Central Processing of Itch in the Midbrain Reward Center. Neuron 2019, 102, 858-872.e5. doi:10.1016/j.neuron.2019.03.030.

14. Wahlgren CF, Hägermark O, B.R. Patients' perception of itch induced by histamine, compound 48/80 and wool fibres in atopic dermatitis. Acta Dermato-Venereologica 1991, 71, 488-494.

15. Lu, Y.; Wong, T.T.W.; Chen, F.; Wang, L. Compressed Ultrafast Spectral-Temporal Photography. Physical Review Letters 2019, 122. doi:10.1103/physrevlett.122.193904.

16. Umeda, K.; Noro, Y.; Murakami, T.; Tokime, K.; Sugisaki, H.; Yamanaka, K.; Kurokawa, I.; Kuno, K.; Tsutsui, H.; Nakanishi, K.; Mizutani, H. A novel acoustic evaluation system of scratching in mouse dermatitis: Rapid and specific detection of invisibly rapid scratch in an atopic dermatitis model mouse. Life Sciences 2006, 79, 2144-2150. doi:10.1016/j.lfs.2006.07.010.

17. Inagaki, N.; Igeta, K.; Shiraishi, N.; Kim, J.; Nagao, M.; Nakamura, N.; Nagai, H. Evaluation and Characterization of Mouse Scratching Behavior by a New Apparatus, MicroAct. Skin Pharmacology and Physiology 2003, 16, 165-175. doi:10.1159/000069755.

18. Cambridge-In-Colour. Understanding Camera Autofocus. https://www.cambridgeincolour.com/ tutorials/camera-autofocus.htm, 2019. Accessed December 4, 2019. 
261 19. Elliott, G.; Vanwersch, R.; Bruijnzeel, P. An automated method for registering and quantifying 262 scratching activity in mice. Journal of Pharmacological and Toxicological Methods 2000, 44, 453-459. 263 doi:10.1016/s1056-8719(01)00111-3.

264 20. Dong, X.; Dong, X. Peripheral and Central Mechanisms of Itch. Neuron 2018, 98, $482-494$. 265 doi:10.1016/j.neuron.2018.03.023. 